\title{
ABLATION OF ABNORMAL CORTEX IN CEREBRAL PALSY*
}

BY

\section{WILDER PENFIELD}

\section{From the Department of Neurology and Neurosurgery, McGill University, and the Montreal Neurological Institute}

Cerebral palsy may be defined, in a broad sense, as loss of function due to brain injury. Such injury may affect motor centres producing spastic paralysis of an arm or leg, but it may also affect other areas of the brain and thus cripple other functions than those of movement of the arm and leg.

Hemispherectomy has been proposed recently as a method of treatment of infantile hemiplegia by Krynauw (1950) followed by Cairns (1951). The claim made is (1) that cerebral seizures may be stopped, (2) the mental state improved, and (3) motor function facilitated because of a lessening of spasticity in the paretic limbs. In the 12 cases of severe hemiplegia reported by Krynauw this very radical procedure was apparently well justified. The question to be decided is whether less radical ablations may not be preferable. The results reported for these hemispherectomies illustrate the truth of the thesis, to which I have subscribed for some time, that abnormal cortex, incapable of useful service, does, in some cases, exert a noxious influence upon the rest of the brain which might otherwise function normally Let us examine this thesis under the headings of epilepsy, mental retardation, and spasticity.

\section{Epilepsy}

For some years we have been in the habit of excising abnormal cerebral cortex in the treatment of focal epilepsy. To obtain good results wide excision is often necessary but in the great majority of cases there is normal as well as abnormal cortex in that hemisphere. It seems reasonable that the bad should be sacrificed and the good spared for future useful function.

In the treatment of epilepsy, periods of five to 10 years should be allowed to elapse before a summary and conclusion is made of a series of adequate size. We have in the past made three follow-up studies of the results of surgical ablation of focal atrophic epileptogenic lesions of the cortex

* Read before the American Academy for Cerebral Palsy, Boston, on November 16, 1951. in the years ending 1939,1944 , and 1950 , in a total of 234 patients.

The first study, which was carried out with Theodore Erickson, included all cases of this type operated upon at the Montreal Neurological Institute between 1929 and 1939 (115 patients). They were followed up from one to 11 years. In this series $43 \%$ were placed in the success group, which means that each case either had no postoperative attacks, or had one or two attacks before epilepsy ceased. The second study, carried out with Harry Steelman, covered the period 1939-44 (Penfield and Steelman, 1947) with a follow-up period of one to seven years in 59 cases of cortical excision with an operative mortality of $1.2 \%$. Of these, $55.8 \%$ were placed in the success group : that is to say, about half of this group had had no attacks after operation and the other half had two or three attacks before cessation.

The third report, carried out in 1950 with Herman Flanigin, consisted in a follow-up of 60 cases of radical operation for temporal lobe epilepsy (psychomotor epilepsy of Gibbs and Lennox). The results were about the same, $55 \%$ of undoubted success.

Most of the patients in each series were adults, but it may be pointed out that of the 59 cases reported in the second series, 17 patients were suffering from seizures produced by an unquestioned birth injury and the attacks usually began in childhood. (In many other cases birth compression was a possible cause of the lesions found.) The incidence of success as the result of operation rose in these 17 cases from the general average of $56 \%$ to $76 \%$. Thus it may be seen that birth injury provided us with our most favourable group. A single example is given below.

Case 1.-M. M. was referred by Dr. A. Jimenez, Bogota. This little girl, aged 7 years, had received a severe brain injury at birth. She had left hemiparesis and hyperactive deep reflexes and plantar extensor response on the left side. There was marked loss of cortical discriminatory sensibility on the left and smallness of the left arm, leg, and trunk. Her disability in arm and leg was not as great as might be expected from complete destruction of the motor cortex. 


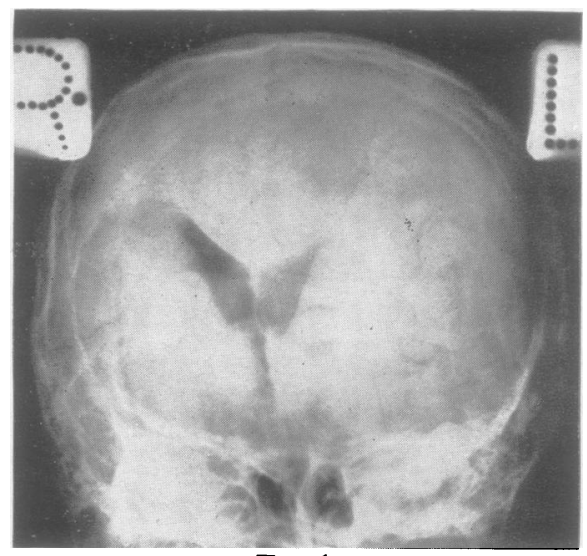

FIG. 1

FIG. 1.-Prneumoencephalogram (Case 1) shows the right cranial chamber to be small and the total volume of the right hemisphere to be very much smaller than the left.

Fig. 2.-Cortical destruction in right hemisphere in Case 1. Note subdural adhesions, also small gyri at periphery of lesion seen dimly through the thickened arachnoid membrane.

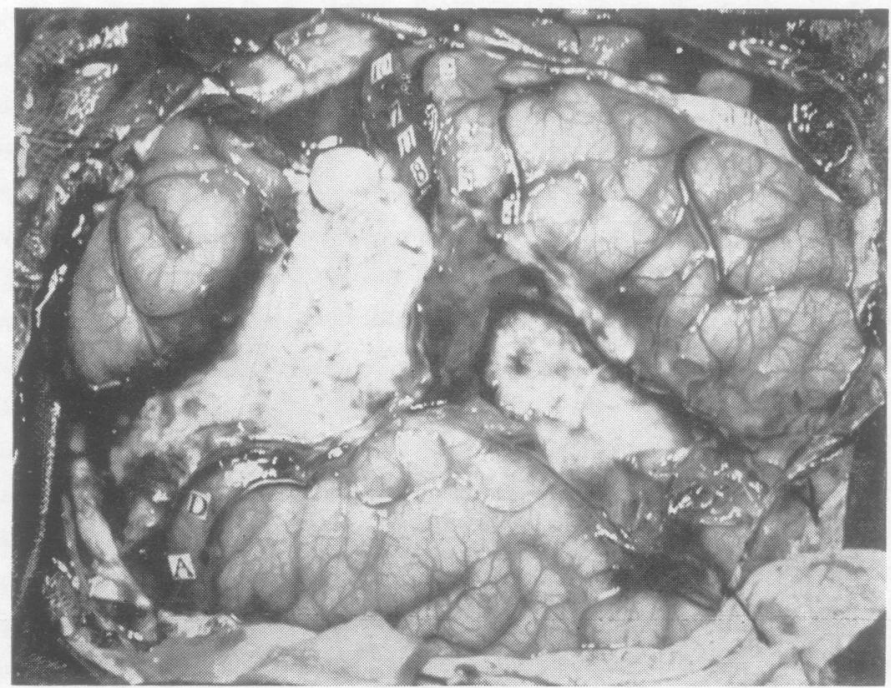

Fig. 3

Cerebral seizures had begun at the age of $4 \frac{1}{2}$. She had been well behaved and intelligent, but during the year before operation her mother was of the opinion that she had been "slowing down".

Pneumoencephalography showed marked smallness of the right hemisphere (Fig. 1). At the time of right osteoplastic craniotomy there was a central area of brain destruction surrounded by a peripheral zone of shrivelled gyri (Fig. 2).

Electrocorticography demonstrated a focus of spike discharges in some of these gyri located in the postcentral region. After careful excision of all abnormal gyri down to the wall of the ventricle (Fig. 3) the remaining cortex appeared normal. The spike discharges were gone, and it is of interest that with them a sharp wave E.E.G. focus that had been present in the temporal lobe (at A and D, Fig. 3) also disappeared.

This case illustrates the fact that a small hemisphere may contain normal cortex and abnormal cortex as well as areas of complete destruction. The removal involved parts of the parietal, temporal, and frontal lobes but spared the arm and leg area of the precentral gyrus, as is shown in Fig. 3. (Case 1) and abnormal yri have been removed by suction down to Rolando. Note the normal width of remaining gyri. intravenous pentothal was used to induce sleep during the opening. Child awake and cooperative during cortical stimulation and electrocorticography. Stimulation at and 11 produced movement of sition of thumb and finger. $A$ and $D$ indicate sharp wave E.E.G. focus which disappeared following the ablation. 
The little girl has had no increase in hemiparesis and no seizures in the year that has followed operation.

\section{Mental Retardation}

When abnormal brain is removed in the treatment of cerebral seizures there results, not infrequently, a marked improvement in mental capacity and behaviour. This is usually true when the attacks have been successfully stopped or greatly reduced. Of course the psychological effect of freedom from seizures may be a factor in this improvement, a factor which applies to adults but hardly to children. The decrease in the amount of anticonvulsant drugs may also be a feature, but phenobarbital alone is invariably continued during the first few postoperative years.

Progressive mental retardation is a prominent feature of some cases and is absent in others, even though seizures may be frequent in both. This would suggest that the abnormal area of brain exerts a noxious influence upon normal areas even in the absence of overt seizures.

A study of the effect of removal of abnormal brain was carried out by Hebb and Penfield (1940). Extensive removal of both anterior frontal lobes was carried out in a young man to stop seizures. The primary objective was only partially achieved, as he had two seizures in the subsequent 15 months.

Professor Donald Hebb carried out exhaustive psychological studies and concluded that, even though about one-third or more of each frontal lobe was missing after operation, "there was a striking post-operative improvement in personality and intellectual capacity with the same medication as before operation".

The conclusion was not that a normal individual with normal brain may be improved by cortical ablation, but that " abnormal areas of brain may produce, in a positive sense, both decreased mental capacity and abnormalities of behaviour, and that the absence of these areas may allow other parts of the brain to recover their normal functions".

Electroencephalography also gives evidence that in such cases partly destroyed cortex exerts an influence on otherwise normal areas of the brain. Diffuse abnormality of electrical potentials may appear in both hemispheres after a period of time, especially in the case of those patients who are said to be clinically retrogressing. Dr. Herbert Jasper has concluded from post-operative study of numerous cases in our clinic that diffuse and continuous E.E.G. abnormality sometimes disappears or may be greatly reduced after successful ablation of a focal epileptogenic area of cortex in one hemisphere. This is seen most often in children.
There are young children and infants who have received brain damage due to ischaemia or trauma at birth, but who seem to do well for a period. Then cerebral seizures appear and their intelligence fails or development is arrested. There are such patients who become increasingly hemiplegic as the attacks continue. Every clinician of any experience must have seen this sad retrogression more than once. For lack of better explanation he may make a presumptive diagnosis of chronic encephalitis or degenerative disease of the brain.

The patient reported below was studied over an eight-year period and reported by Penfield and Livingston (1949).

Case 2.-The birth of $\mathrm{H}$. F. was not considered unusual. On the second day of life, however, he vomited and had two short convulsions. The spinal fluid contained blood. From the fifth day onward, he seemed normal, held up his head at 3 weeks, sat alone at 4 months, and appeared to have normal intelligence.

At 5 months he had a left-sided convulsion, and similar attacks followed with increasing frequency despite medication. At 1 year he was unable to sit up and seemed completely disassociated from his surroundings.

An electroencephalogram showed marked abnormality on both sides (Fig. 4a) with epileptogenic activity on the right. Operation was carried out on the right, and cortex that had been partly destroyed by natal intracerebral haemorrhage was removed. Six weeks later the E.E.G. was normal as shown in Fig. 4B. At 8 years of age the boy was free of attacks. He was in the second grade at school and had a mental age of 6 years and 2 months which was far better than anything that could have been hoped for at 12 months without operation.

\section{"Nociferous" Cortex}

Let us consider the case of any patient who has a brain lesion and is destined to develop epileptic seizures. In the period between the reception of brain damage and the onset of seizures, it is obvious that a gradual alteration occurs in injured grey matter. -... This interstitial " or - cellular alterationeventually results in seizures years, perhaps, after the lesion was established.

The nature of the change in the damaged area of grey matter is not clear. But Pope, Morris, Jasper, Elliot, and Penfield (1947) have demonstrated elevated cholinesterase content in such foci, and Tower and Elliot (1952) have shown that there is a local decrease in the capacity to combine acetylcholine. The electroencephalogram suggests that this is associated with increased spontaneous liberation of energy in the area.

It would appear then that that focus of epileptogenic activity may interfere with the function and 


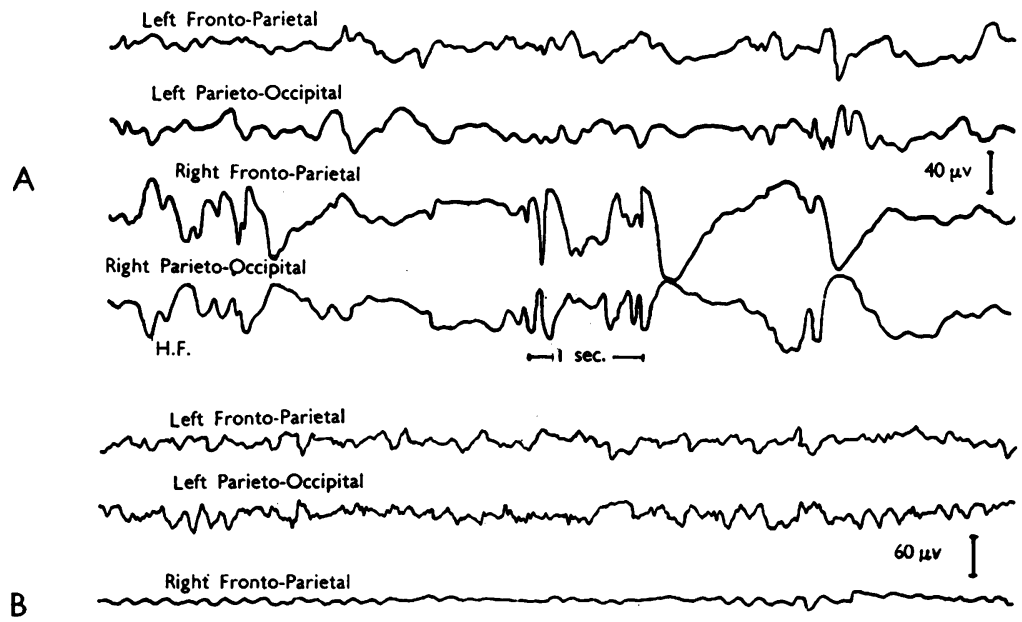

Fig. 4.-Electroencephalograms of Case 2.

A. Before operation : note random delta and sharp waves arising from all head regions, with phase reversal of spikes, sharp waves and random delta waves occurring midway between right ear and vertex.

B. Six weeks after ablation of epileptogenic area of cortex in right frontal lobe near falx. This record shows only a background rhythm at a frequency of 4 to 5 per second. This should be considered normal for a child of this age. (Interpretation by Professor Herbert Jasper.)

B

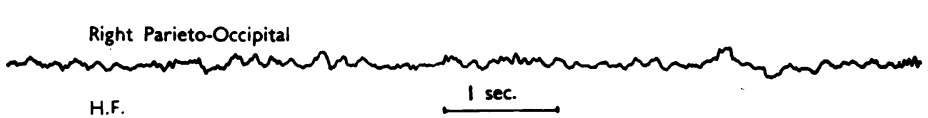

the evolution of the uninjured portions of the brain. What begins as a local affair ends with generalized abnormality. Such an area of cortex must be considered as a source of noxious influences, a concept which might be described by the somewhat obsolete adjective " nociferous".

When such a train of events is established in infancy and the child arrives at years which should be years of understanding, there may be little left to save. Operative procedures are better controlled and less subject to risk at 10 years of age than at 2 , but we have been forced to reconsider our policy of postponement in regard to radical ablation of epileptogenic cortex in those cases in which there is evidence of progressive intellectual degeneration or progressive paralysis.

Does mental retardation always develop in patients who have recurring seizures due to a cortical lesion? Patients often ask this question. The answer must be, "No, by no means." In some there is intellectual deterioration; in others there seems to be little or none. But when such change begins, the outlook for the future is ominous.

In regard to localization it may be said that temporal lobe seizures are most often associated with progressive intellectual and behavioural abnormality in adults. Among infants and children no particular localization has been apparent. The number of overt seizures is often no index of whether or not a lesion of the brain is exerting a noxious influence upon its general performance.

At times very large excisions are necessary when the area of partly destroyed cortex is large and when electrographic abnormality is widespread. Such a situation may be illustrated by the following :

Case 3 : K. M.-The extent of removal which seemed necessary is shown by the broken line in Fig. 5. Fig. 6 shows that the posterior half of the right hemisphere has been removed and that the remaining cortex is comparatively normal. The ventricle is about to be closed.

\section{Motor Function}

We have gradually come to realize that sufferers from severe hemiplegia are sometimes benefited by complete ablation of the abnormal Rolandic sensori-motor cortex. This apparent paradox is to be explained by the fact that a lesion of one hemisphere may render the motor cortex useless and yet not destroy it. Under such circumstances removal of the cortex may result in a decrease of spasticity (Welch and Penfield, 1950).*

In order to understand this, it must be realized that ablation of the precentral motor gyrus of a normal individual does not produce complete paralysis. It produces initial paralysis which is soon replaced by paresis more marked in the arm than in the leg. Such an individual can walk and he can use his arm and hand for gross movements. Skilled movements of the fingers and movements of individual toes are lost forever. Thus a patient, who already has no control of the hand for delicate movements, has little to fear as the result of excision of the arm area, and it is possible that the arm may

* It may also result in cessation of Jacksonian seizures, supposing, of course, that the patient had complained of seizures. 


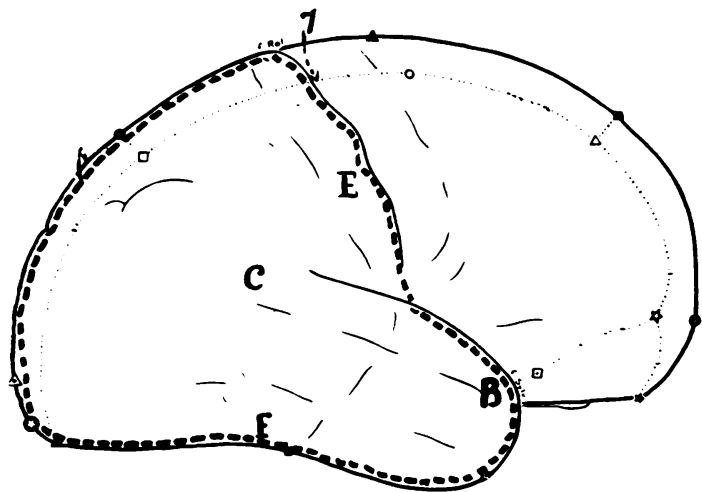

FIG. 5.-The extent of cortex removed in Case 3 is indicated by the broken line, and the extent of electrocorticographic abnormality by letters E, C, F, and B. Metrazol was given intravenously during operation to increase the abnormal potentials.

FIG. 6.-Occipital, parietal, and temporal lobes have been removed in Case 3. Stimulation at 7 produced movement of left leg. The ventricle is open but enough of the ependyma has been left intact so that it can be closed by silk sutures, the first of which is shown. This closure avoids post-operative elevation of temperature (" aseptic meningitis").

thus be freed of a good deal of the spasticity that hampers those movements of which he is otherwise capable.

After the motor cortex is excised movement seems to be controlled by subcortical mechanisms (Penfield and Rasmussen, 1950). A lesion deep in one hemisphere results in a much more severe paralysis than that produced by cortical excision.

On the other hand there are many cases of cerebral palsy in which ablation of motor cortex will convert hemiparesis into a more severe disability. In such cases, unless the motor gyrus serves as epileptogenic focus, it is preferable to spare this area of cortex.

One more case may be cited very briefly, that of a baby who was treated with very radical operation. It is possible that Mr. Krynauw would have recommended hemispherectomy. The result is encouraging so far, but only time and a great deal more experience will make it clear how radical the neurosurgeon should be.

Case 5.-F. S. was referred by Dr. Henri Gastaut, Marseilles. Birth was difficult

FIG. 7.-Operative exposure of the left hemisphere in Case 5. As the dura was reflected adhesions to the pia had to be cut. Beneath the adhesions the gyri were narrow and indurated. Anteriorly and posteriorly they appeared normal.

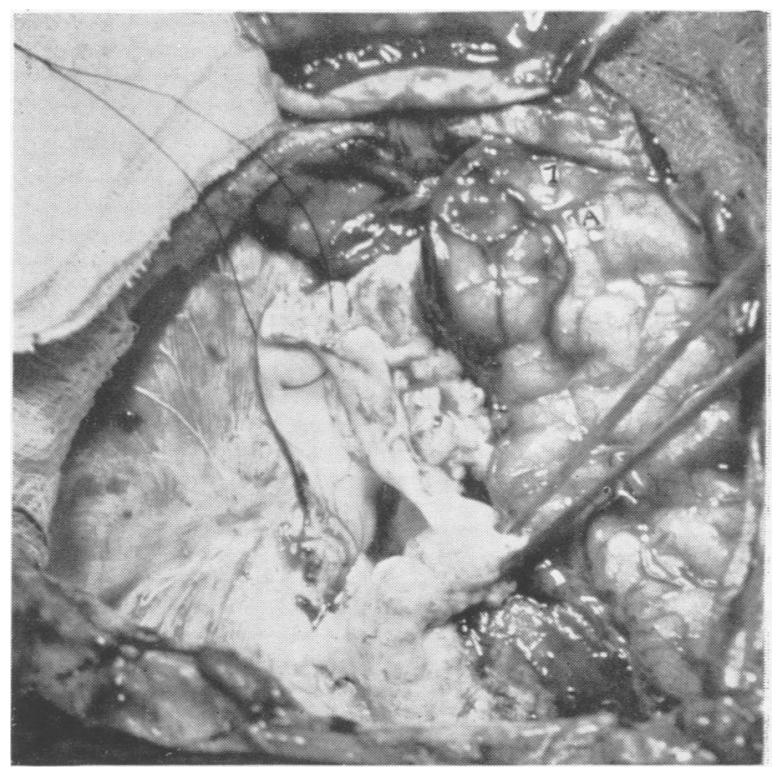

and the child was cyanosed. On the second day of life there were convulsive attacks involving the right arm. At 5 months right-sided seizures appeared. At this time the parents believe the child to have been normal mentally. He ate well, played games such as " patty cake", and handled toys normally.

From 5 months on there was steady retrogression and at $2 \frac{1}{2}$ years, when operation was undertaken, the child was grossly retarded, did not play with toys and was unable to stand or walk alone.

At that period, E.E.G. examinations by Professor Gastaut showed "spikes" and also sharp wave abnor-

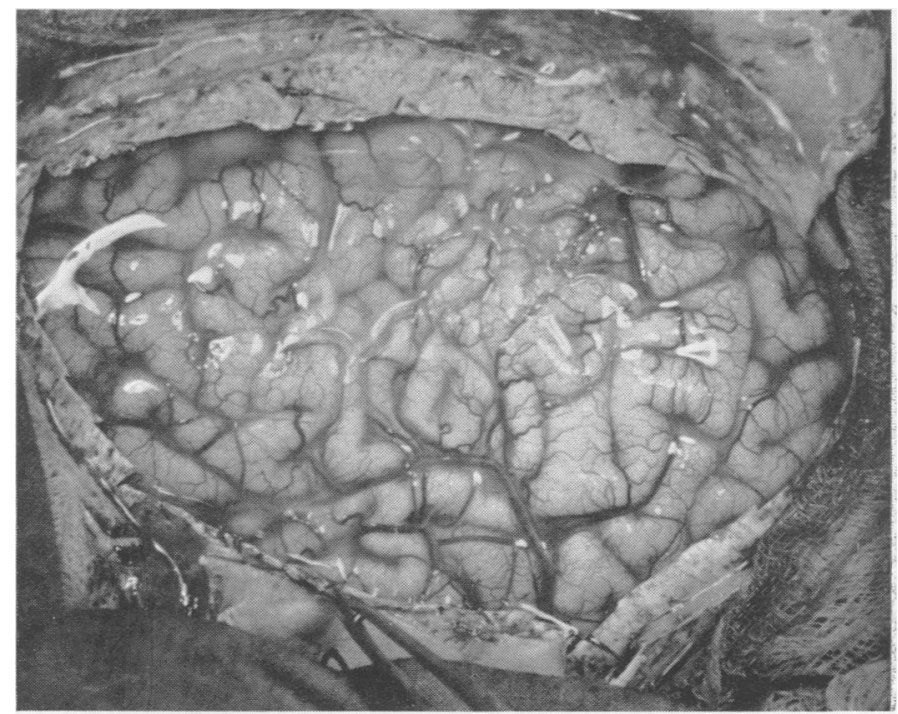


malities from both hemispheres, although earlier electroencephalograms had shown only a focus in the left hemisphere with normal activity on the right.

The boy had a right hemiparesis and a small right arm and leg. Radiographs showed comparative smallness of the left cranium. The ventricles were somewhat large, the left being larger than the right, particularly in the central region (portions 2 and 3 ).

A large left-sided osteoplastic craniotomy was carried out. This disclosed a remarkable zone of small gyri in the central region, some being as narrow as 2 or $3 \mathrm{~mm}$. Over this area the dura was adherent, as shown in Fig. 7. In the frontal and occipital regions there was a gradual change to gyri of relatively normal appearance (Fig. 7).

A wide excision was carried out and this was then enlarged further because Dr. Jasper reported continuing electrocorticographic abnormalities on the frontiers. The final removal is shown in Fig. 8.

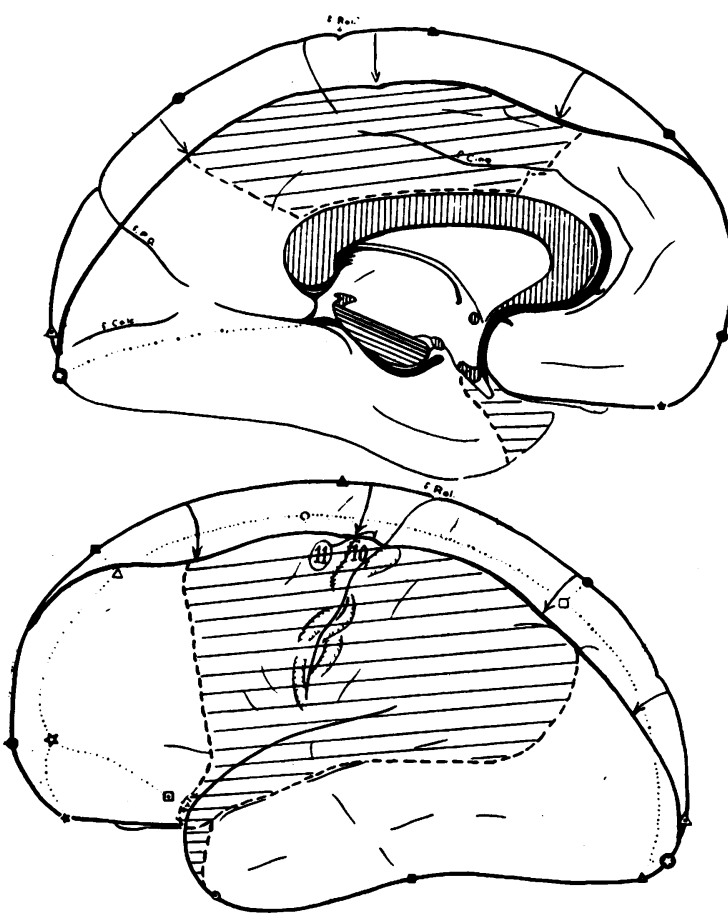

Fig. 8.- The extent of cortical ablation in Case 5 is indicated by the shaded area. It was necessary to include the whole of the first temporal convolution down to and including the uncus. Stimulation at point 10 produced arm movement and at 11 movement of foot.

The/ smallness of the left hemisphere compared with its expected size is indicated by the arrows to the inner outline.
A year after operation the child had apparently had no seizures. The electroencephalogram was very much better. He used his leg better than before operation, although it was weak. The right arm was carried in flexion. There was "magnificent" psychological improvement and he spoke in monosyllables.

\section{Summary}

In conclusion, patients who suffer from cerebral palsy may be benefited by cortical excision in various ways. But they must be very carefully studied and selected before such radical procedures are to be considered.

The possible benefits may be reviewed as follows. (1) The patient who is subject to recurring cerebral seizures may be relieved of his attacks. (2) The patient who has a discharging lesion of the cortex and shows progressive mental retardation may be greatly improved by excision of the partially destroyed cortex provided that the intellectual degradation has not gone too far. This applies particularly to children in the early stages of mental retardation and behaviour abnormality. (3) Patients suffering from severe hemiplegia from infancy may be freed from spasticity to a considerable extent by cortical motor excision.

In our experience, complete hemispherectomy would rarely be advisable. But subtotal hemispherectomy and the discriminating ablation of all nociferous cortex is a form of therapy that can bring great improvement to selected sufferers from cerebral palsy.

\section{BibliograPHY}

Cairns, H. (1951). Lancet, $2,411$.

Hebb, D. O., and Penfield, W. (1940). Arch. Neurol. Psychiat., Chicago, 44, 421.

Krynauw, R. A. (1950). Journal of Neurology, Neurosurgery and Psychiatry, 13, 243.

Penfield, W., and Flanigin, H. (1950). Arch. Neurol. Psychiat., Chicago, 64, 491.

_- , and Livingston, S. (1949). Pediatrics, 4, 157.

--, and Steelman, H. (1947): Ann. Surg., 126, 740.

_, and Rasmussen, T. (1950) "The Cerebral Cortex of Man." New York.

Pope, A., Morris, A. A., Jasper, H., Elliot, K. A. C., and Penfield, W. (1947). In "Epilepsy." Res. Publ. Ass. nerv. ment. Dis. (1946), 26, 218.

Tower, D. B., and Elliot, K. A. C. (1952). J. appl. Physiol. In the press.

Welch, K., and Penfield, W. (1950). J. Neurostırg. $7,414$. 